\title{
W019
}

\section{Subsurface Offset Behaviour in Velocity Analysis with Extended Reflectivity Images}

\author{
W.A. Mulder* (Shell Global Solutions International BV / Delft University of \\ Technology)
}

\section{SUMMARY}

Migration velocity analysis with the wave equation can be accomplished by focusing of extended migration images, obtained by introducing a subsurface offset or shift. A reflector in the wrong velocity model will show up as a curve in the extended image. In the correct model, it should collapse to a point.

The usual approach to obtain a focused image involves a cost functional that penalizes energy in the extended image at non-zero shift. Its minimization by a gradient-based method should then produce the correct velocity model. Here, asymptotic analysis and numerical examples show that this method may be too sensitive to amplitude peaks at large shifts at the wrong depth and to artifacts. A more robust alternative is proposed that can be interpreted as a generalization of stack power and maximizes the energy at zero subsurface shift. A real-data example is included. 


\title{
Subsurface offset behaviour in velocity analysis with extended reflectivity images
}

\author{
W.A. Mulder \\ Shell Global Solutions International BV \& TU Delft
}

\section{Summary}

Migration velocity analysis with the wave equation can be accomplished by focusing of extended migration images, obtained by introducing a subsurface offset or shift. A reflector in the wrong velocity model will show up as a curve in the extended image. In the correct model, it should collapse to a point.

The usual approach to obtain a focused image involves a cost functional that penalizes energy in the extended image at non-zero shift. Its minimization by a gradient-based method should then produce the correct velocity model. Here, asymptotic analysis and numerical examples show that this method may be too sensitive to amplitude peaks at large shifts at the wrong depth and to artifacts. A more robust alternative is proposed that can be interpreted as a generalization of stack power and maximizes the energy at zero subsurface shift. A real-data example is included.

Topics: 25 Velocity Model Building; 04 Full Waveform Seismic Inversion

Main objectives: Improve an algorithm for automatic velocity analysis with the full wave equation

New aspects covered: Asymptotic analysis of subsurface offset behaviour, formulation of a robust cost functional for optimization, real-data example 


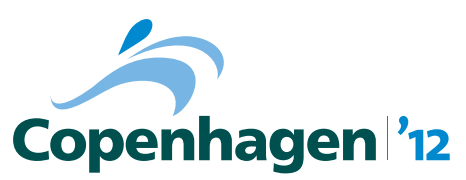

\section{Introduction}

Precise subsurface imaging with seismic data requires an accurate velocity model. One approach to migration velocity analysis with the wave equation is to build extended migration images with a subsurface spatial (MacKay and Abma, 1992; Rickett and Sava, 2002; Biondi and Sava, 2004a,b) or temporal shift (Faye and Jeannot, 1986; Sava and Fomel, 2006; Brown et al., 2008; van Leeuwen and Mulder, 2010) and focusing them at zero shift (Shen et al., 2003, 2005; Mulder, 2008; Symes, 2008). The spatial or temporal shift can be interpreted as action at distance and compensates velocity errors. Because reflectors may be observable in the extended image as curved events, even with the wrong velocity, it is easier to improve their focusing then for instance with stacking power. With the latter, if the velocity model is too far away from the correct one, there is nothing that stacks, so nothing to use for velocity updating. In practice, the focusing of extended images produces results of mixed quality. Sometimes, the method works very well, sometimes it has difficulties. The data should be free of multiples, otherwise, the method may converge to the wrong model (Mulder and van Leeuwen, 2008). Point scatterers and diffractions may cause problems (Vyas et al., 2010). Artifacts in the extended images may lead gradient-based optimization methods astray.

To improve the robustness of the method, a modification of the focusing cost functional is proposed. Asymptotic analysis and numerical tests show that the usual penalization of energy at non-zero shift may give too strong a bias to amplitude peaks at large shifts at the wrong depth and to artifacts.

\section{Cost function}

The extended reflectivity is defined as

$$
R(\mathbf{x} ; \mathbf{h})=\operatorname{Re} \sum_{s, \omega} \omega^{2} p_{s}^{*}\left(\mathbf{x}-\frac{1}{2} \mathbf{h}, \omega\right) q_{s}\left(\mathbf{x}+\frac{1}{2} \mathbf{h}, \omega\right) .
$$

The wavefield $p_{s}(\mathbf{x}, \omega)$ is generated by a source with index $s$, whereas $q_{s}(\mathbf{x}, \omega)$ is the reverse-time wavefield due to the observed data at the receivers that correspond to this source. To avoid a data explosion, we can restrict the choice of $\mathbf{h}$ to a single coordinate direction. The cost function $J=J_{x}+\gamma J_{z}$ for migration velocity analysis with horizontal subsurface shift in two space dimensions can be chosen as the weighted sum of

$$
J_{x}=\frac{1}{2} \sum_{x, z, h_{x}} W(x, z) \psi\left(h_{x}\right)\left|\Xi_{x} R\left(x, z ; h_{x}, 0\right)\right|^{2}, \quad J_{z}=\frac{1}{2} \sum_{x, z, h_{z}} W(x, z) \psi\left(h_{z}\right)\left|\Xi_{z} R\left(x, z ; 0, h_{z}\right)\right|^{2} .
$$

The functional $J_{z}$ may be necessary in the presence of steep dips (Biondi and Shan, 2002). The filters $\Xi_{x}$ and $\Xi_{z}$ should remove long-wavelength components and events with large or small dip, respectively, as proposed earlier (Mulder, 2008). Weibull (2011) introduced a simpler but possibly more noise-sensitive filter, involving the first derivative in the orthogonal direction. The function $\psi\left(h_{x}\right)$ is commonly chosen as $h_{x}^{2}$, but will be changed here. $W(x, z)$ is a spatial weighting function.

\section{Asymptotic analysis}

To study the behaviour of the extended reflectivity, I consider a single horizontal reflector in a constant velocity model for a marine acquisition with positive surface offsets in the high-frequency limit using the stationary phase approximation. For a delta-function reflector at depth $z_{0}$, the observed data are $p^{\text {obs }}\left(\omega, \hbar_{0}\right)=A_{0} \exp \left[2 i k_{0} r_{0}\right]$, where $r_{0}=\left(\hbar_{0}^{2}+z_{0}^{2}\right)^{1 / 2}$, with amplitude $A_{0}^{2 \mathrm{D}}=\frac{(i-1)}{8 z_{0}} \sqrt{\frac{\omega c_{0}^{3} r_{0}}{2 \pi}}$ in $2 \mathrm{D}$ or $A_{0}^{3 \mathrm{D}}=$ $\frac{i \omega c_{0}}{16 \pi z_{0}}$ in $3 \mathrm{D}$ as a function of surface half-offset $\hbar_{0}$ for a source and receiver at zero depth and for a constant velocity $c_{0}$ and wavenumber $k_{0}=\omega / c_{0}$. Note that in $2 \mathrm{D}$, the amplitude increases with offset. If we consider only a horizontal subsurface shift $h_{x}=2 \hbar_{x}$, the stationary phase approximation shows that the extended reflectivity in a model with constant velocity $c \neq c_{0}$ follows the curve

$$
z=z_{c}=\gamma \sqrt{z_{0}^{2}-\beta \hbar_{0}^{2}}, \quad \hbar_{x}=-\beta \hbar_{0}
$$


where $\gamma=c / c_{0}$ and $\beta=\gamma^{2}-1>-1$. If $\beta>0$, the curve truncates at $z_{0}=\hbar_{0} \sqrt{\beta}$ or $\hbar_{x}=-z_{0} \sqrt{\beta}$, assuming $\hbar_{0}>0$. Note that the surface and subsurface offsets are related via $\beta$, which depends on the velocity error. The amplitude along the curve (1) is

$$
A^{2 \mathrm{D}}=\frac{(1+i \operatorname{sign} \beta) \gamma^{5}}{128 z_{0} z_{c}^{2}} \sqrt{\frac{2 \pi c_{0}^{9} r_{0}^{5}}{|\beta|}}, \quad A^{3 \mathrm{D}}=\frac{(1+i \operatorname{sign} \beta)(1+i) \gamma^{3} c_{0}^{3} r_{0}}{512 \pi z_{0} z_{c}^{2} \sqrt{|\beta|}} .
$$

This stationary-phase estimate assumes an infinite acquisition. Truncation at the shortest or longest offset yields a curve $z=\sqrt{\gamma^{2}\left(\hbar_{0 m}^{2}+z_{0}^{2}\right)-\left(\hbar_{x}-\hbar_{0 m}\right)^{2}}$, where $\hbar_{0 m}$ denotes either the shortest or longest surface half-offset.

\section{Numerical results}

Figure 1 shows the curves (a) and amplitude (b) along the main curve for a velocity of $1.6 \mathrm{~km} / \mathrm{s}$ using data based on $1.5 \mathrm{~km} / \mathrm{s}$ and a horizontal reflector at $750 \mathrm{~m}$ depth for the 2-D case. Offsets ranged from 10 to $4000 \mathrm{~m}$ with a $10-\mathrm{m}$ interval in a marine-type acquisition (positive offsets to the right of the source) and shots ran from -3000 to $3000 \mathrm{~m}$ at a $25-\mathrm{m}$ interval. The dash-dotted and dashed curves are caused by truncation of the acquisition at shortest and longest offsets. Their amplitudes may be reduced by a suitable offset taper that smoothly decreases the data amplitudes towards the endpoints of the seismic line and the shot range. The amplitude in Fig. 1(b) increases with subsurface offset until it truncates at $\hbar_{x}=$ $-z_{0} \sqrt{\beta}$. The curve obtained from the numerical simulation continues beyond with a smaller amplitude, due to the truncation of the surface offset range, and eventually will vanish. The differences between the numerical and asymptotic results near the minimum and maximum offsets are most likely caused by the finite ranges for frequency, shot and offset. The truncation of the stationary phase integral provides additional contributions at the endpoints that were not included in the asymptotic estimates. Figure 2 shows similar results for a velocity of $1.4 \mathrm{~km} / \mathrm{s}$. At the correct velocity, the curve $z=z_{c}$ collapses to a point and only the endpoint contributions of the truncated acquisition remain. The figures demonstrate that the peak amplitude occurs away from zero subsurface offset in the wrong velocity model and at a depth that can be far away from the true depth $z_{0}$. Penalizing this peak by a weighting function $\psi=\psi_{1}\left(h_{x}\right)=h_{x}^{2}$ will give a contribution to the gradient that should update the velocity model at a depth that may be far off.

Figure 3(a) shows that the peak amplitude may also increase as a function of the velocity error $c / c_{0}-1$, using constant values for both $c$ and $c_{0}$. Therefore, an alternative weighting function is proposed that is less sensitive to large amplitudes far away. Instead of minimization, the cost function is maximized with $\psi_{2}\left(h_{x}\right)=1 /\left[1+\left(h_{x} / \alpha_{x}\right)^{2}\right]^{p}$ with, for instance, $p=\frac{1}{2}$ or $p=1$. The power $p$ should be large enough too reduce the sensitivity to amplitudes and artifacts at large $h_{x}$, but not so large that the convexity of the functional is destroyed. In the limit for small $\alpha_{x}$, this cost function starts to resemble stack or imaging power maximization (Toldi, 1989; Soubaras and Gratacos, 2007; van Leeuwen and Mulder, 2010). Figure 3(b) displays scans of the cost functional $J_{1}$ that is based on minimization with $\psi_{1}\left(h_{x}\right)=h_{x}^{2}$ and $J_{2}$ based on maximization with the proposed weighting function $\psi_{2}$. Note that $J_{1}$ is more convex but does not have its minimum at the correct model. The convexity of $J_{2}$ can be controlled by $\alpha_{x}$.

\section{Real-data example}

The method was applied to a marine data set with a 3-km maximum offset. The 2-D frequency-domain code (Mulder and Plessix, 2004) used frequencies from 6 to $16 \mathrm{~Hz}$ at a $0.25-\mathrm{Hz}$ interval. The velocity model was represented by linear splines with spacings of $200 \mathrm{~m}$ in the horizontal and $20 \mathrm{~m}$ in the vertical direction, whereas the computational grid had a $10-\mathrm{m}$ spacing. The inversion was started with a oneparameter search for the gradient of a velocity model linear in depth that provided the largest functional $J_{2, x}$. After that, gradient-based optimization with a limited-memory BFGS method with bounds (Byrd et al., 1995) was carried out. Figure 4 shows the resulting velocity model after 41 iterations, together with the migration image $R(x, z ; 0,0)$ and a section of the extended reflectivity at $x=2 \mathrm{~km}$. The events 


\section{Copenhagen '12}

in the latter are reasonably well focused, although not everywhere. Also, some artifacts are visible.

\section{Conclusions}

The analysis of amplitudes in extended migration images had led to an alternative, more robust formulation of the cost functional, maximizing energy at zero subsurface offset rather than penalizing amplitudes at non-zero subsurface offsets. The approach generalizes stack power maximization.

\section{References}

Biondi, B. and Sava, P. [2004a] Wave-equation migration velocity analysis. I. Theory. Geophysical Prospecting, 52(6), 593-606, doi:10.1111/j.1365-2478.2004.00447.x.

Biondi, B. and Sava, P. [2004b] Wave-equation migration velocity analysis. II. Subsalt imaging examples. Geophysical Prospecting, 52(6), 607-623, doi:10.1111/j.1365-2478.2004.00448.x.

Biondi, B. and Shan, G. [2002] Prestack imaging of overturned reflections by reverse time migration. SEG Technical Program Expanded Abstracts, 21(1), 1284-1287, doi:10.1190/1.1816889.

Brown, M.P., Higginbotham, J.H. and Clapp, R.G. [2008] Velocity model building with wave equation migration velocity focusing analysis. SEG Technical Program Expanded Abstracts, 27(1), 3078-3082, doi: 10.1190/1.3063984.

Byrd, R.H., Lu, P., Nocedal, J. and Zhu, C. [1995] A limited memory algorithm for bound constrained optimization. SIAM Journal on Scientific Computing, 16(5), 1190-1208, doi:10.1137/0916069.

Faye, J. and Jeannot, J. [1986] Prestack migration velocities from focusing depth analysis. SEG Expanded Abstracts, 5, 438-440, doi:10.1190/1.1893053.

MacKay, S. and Abma, R. [1992] Imaging and velocity analysis with depth-focusing analysis. Geophysics, 57(12), 1608-1622, doi:10.1190/1.1443228.

Mulder, W.A. [2008] Automatic velocity analysis with the two-way wave equation. 70th EAGE Conference \& Exhibition, Extended Abstracts, P165, European Association of Geoscientists \& Engineers.

Mulder, W.A. and Plessix, R.É. [2004] A comparison between one-way and two-way wave-equation migration. Geophysics, 69(6), 1491-1504, doi:0.1190/1.1836822.

Mulder, W.A. and van Leeuwen, T. [2008] Automatic migration velocity analysis and multiples. SEG Technical Program Expanded Abstracts, 27(1), 3128-3132, doi:10.1190/1.3063996.

Rickett, J.E. and Sava, P.C. [2002] Offset and angle-domain common image-point gathers for shot-profile migration. Geophysics, 67(3), 883-889, doi:10.1190/1.1484531.

Sava, P. and Fomel, S. [2006] Time-shift imaging condition in seismic migration. Geophysics, 71(6), S200-S217, doi:10.1190/1.2338824.

Shen, P., Symes, W.W., Morton, S., Hess, A. and Calandra, H. [2005] Differential semblance velocity analysis via shot profile migration. SEG Technical Program Expanded Abstracts, 24(1), 2249-2252, doi: $10.1190 / 1.2148164$.

Shen, P., Symes, W.W. and Stolk, C.C. [2003] Differential semblance velocity analysis by wave-equation migration. SEG Technical Program Expanded Abstracts, 22(1), 2132-2135, doi:10.1190/1.1817759.

Soubaras, R. and Gratacos, B. [2007] Velocity model building by semblance maximization of modulated-shot gathers. Geophysics, 72(5), U67-U73, doi:10.1190/1.2743612.

Symes, W.W. [2008] Migration velocity analysis and waveform inversion. Geophysical Prospecting, 56(6), 765790, doi:10.1111/j.1365-2478.2008.00698.x.

Toldi, J.L. [1989] Velocity analysis without picking. Geophysics, 54(2), 191-199, doi:10.1190/1.1442643.

van Leeuwen, T. and Mulder, W.A. [2010] A comparison of seismic velocity inversion methods for layered acoustics. Inverse Problems, 26(1), 015008 (21pp), doi:10.1088/0266-5611/26/1/015008.

Vyas, M., Geco, W. and Tang, Y. [2010] Gradients for wave-equation migration velocity analysis. SEG Technical Program Expanded Abstracts, 29(1), 4077-4081, doi:10.1190/1.3513711.

Weibull, W.W. [2011] Automatic migration velocity analysis using reverse time migration. 73rd EAGE Conference \& Exhibition, Extended Abstracts, B012, European Association of Geoscientists \& Engineers. 


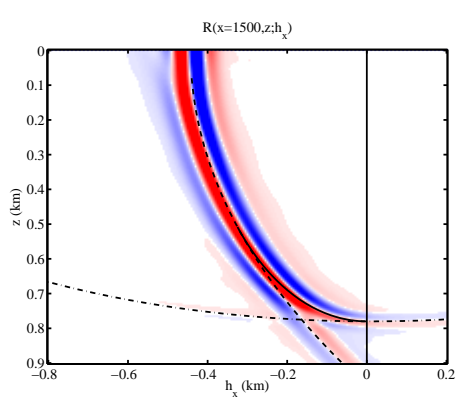

(a)

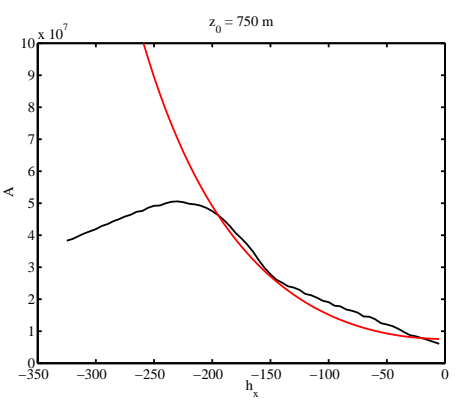

(b)

Figure 1 (a) Extended image at a fixed lateral position $x$ as function of horizontal subsurface offset $h_{x}$ and depth z for too high a velocity of $1.56 \mathrm{~km} / \mathrm{s}$. The dash-dotted line corresponds to the shortest offset, the dashed line to the longest, and the drawn line to the stationary phase curve. The colours show the result of a numerical simulation. (b) Observed amplitude behaviour (black), measured in a window around the stationary e curve, together with the asymptotic result (red), using arbitrary scaling.

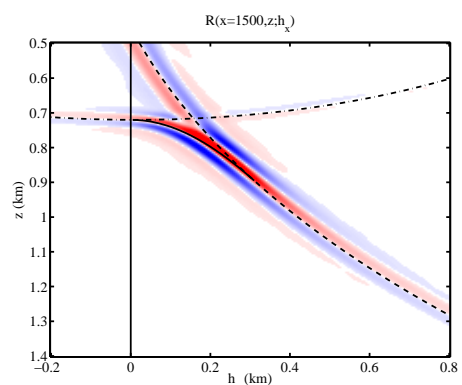

(a)

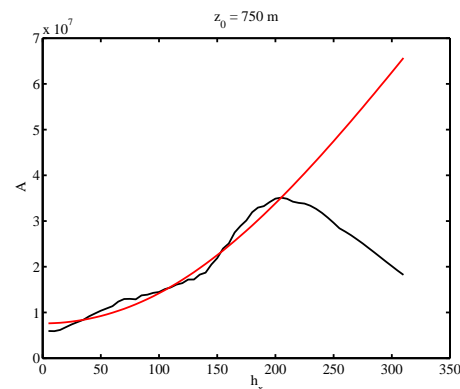

(b)

Figure 2 As Figure 1, but for too low a velocity of $1.44 \mathrm{~km} / \mathrm{s}$.

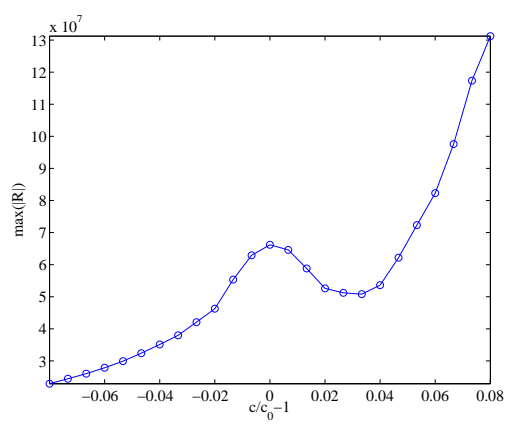

(a)

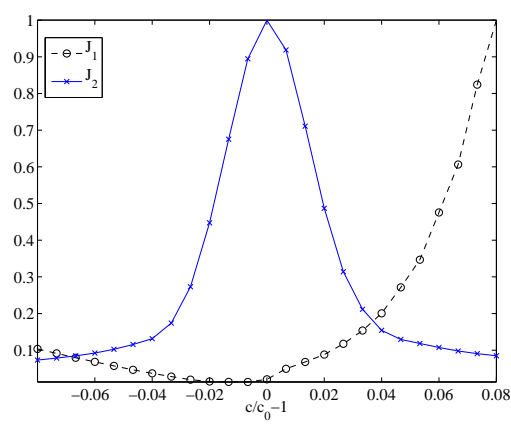

(b)

Figure 3 (a) Observed maximum of $\mid R\left(x, z ; h_{x} \mid\right.$ at $x=1.5 \mathrm{~km}$ for single-reflector data as a function of the velocity error $c / c_{0}-1$. (b) Cost functions as function of the velocity error $c / c_{0}-1 . J_{1}$ (black dashed) was obtained for a weighting function $\psi\left(h_{x}\right)=h_{x}^{2}, J_{2}$ (blue) for $\psi\left(h_{x}\right)=\left[1+\left(h_{x} / \alpha_{x}\right)^{2}\right]^{-1}, \alpha_{x}=100 m$.

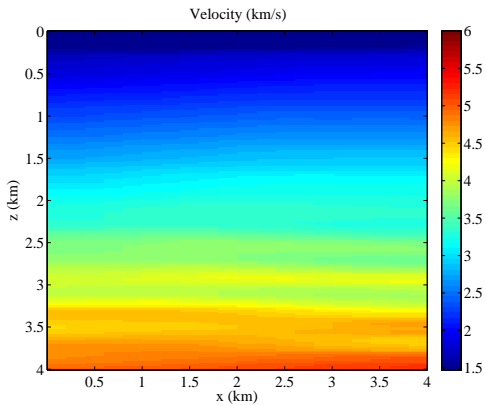

(a)

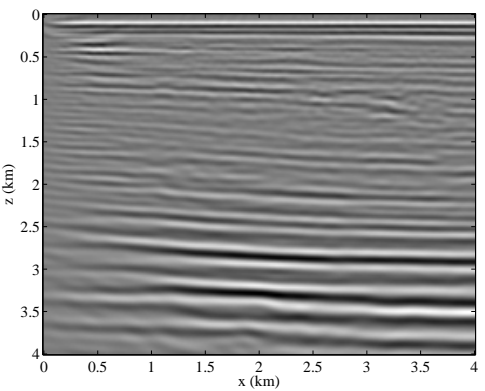

(b)

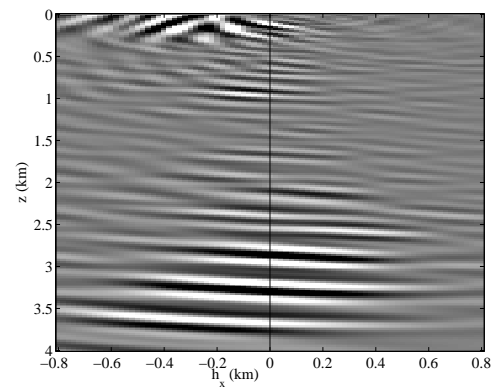

(c)

Figure 4 (a) Velocity model after 41 iterations, (b) migration image, (c) extended image at $x=2 \mathrm{~km}$. 